\title{
The Conceptual Model and Methods of Wave 1 (2016) of the EUREST-PLUS ITC 6 European Countries Survey
}

\author{
Geoffrey T. Fong ${ }^{1,2}$, Mary E. Thompson' ${ }^{1}$ Christian Boudreau', Nicolas Bécuwe ${ }^{3}$, Pete Driezen ${ }^{1}$, Thomas K. Agar', Anne

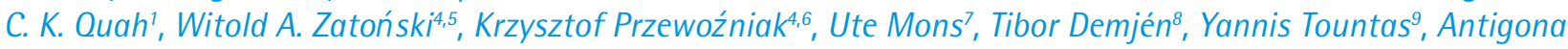 \\ C. Trofor ${ }^{10,11}$, Esteve Fernández ${ }^{12,13}$, Ann McNeill'14,15, Marc Willemsen ${ }^{16}$, Constantine I. Vardavas ${ }^{17,18}$, on behalf of the \\ EUREST-PLUS consortium*
}

\begin{abstract}
Population-level interventions represent the only real approach for combatting the tobacco epidemic. There is thus great importance in conducting rigorous evaluation studies of tobacco control policies and regulations such as those arising from the WHO Framework Convention on Tobacco Control (FCTC) and the European Union's 2014 Tobacco Products Directive (TPD). The ITC 6 European Countries Survey, a component of the Horizon 2020 Project entitled European Regulatory Science on Tobacco: Policy Implementation to Reduce Lung Disease (EUREST-PLUS), was created to evaluate and impact of the TPD in six EU Member States: Germany, Greece, Hungary, Poland, Romania, and Spain. In each country, a cohort survey of a representative national sample of 1000 smokers was conducted. This paper describes the conceptual model, methodology, and initial survey statistics of Wave 1 of the ITC 6E Survey, which was conducted June-September 2016. The ITC 6E Survey's conceptual model, methodology, and survey instrument, were based on the broader 29-country ITC Project cohort studies, which have been conducted since 2002 . The commonality of methods and measures allow a strong potential for cross-country comparisons between the $6 \mathrm{EU}$ countries of the ITC $6 \mathrm{E}$ Project and 3 other EU countries (England, France, The Netherlands) in the ITC Project, as well as the broader set of ITC countries outside the EU.
\end{abstract}

https://doi.org/10.18332/tid/99881

\section{INTRODUCTION}

Given the enormity of the global tobacco epidemic, the most effective approach for reducing its devastation is to create and implement strong interventions at the level of entire populations. The WHO Framework Convention on Tobacco Control (FCTC) is the most prominent of these efforts, given its global reach (with 180 countries and the European Union as FCTC Parties) and the comprehensive set of measures contained within the treaty text, which have been developed to a greater extent through the adoption of guidelines

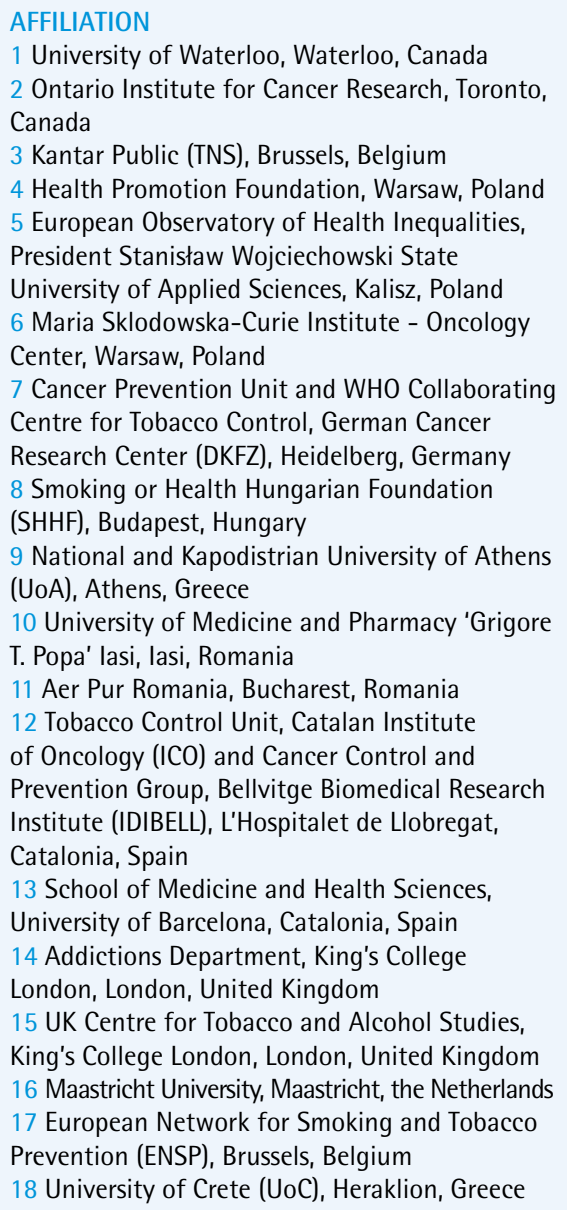

\section{CORRESPONDENCE TO}

Geoffrey T. Fong. Department of Psychology, University of Waterloo, 200 University Avenue West, N2L 3G1 Waterloo, Ontario, Canada.

E-mail: gfong@uwaterloo.ca

KEYWORDS

tobacco control, evaluation, cohort study, EU Tobacco Products Directive, ITC Project

Received: 2 November 2018

Accepted: 20 November 2018 
for a number of policy domains including: price/ tax, smoke-free, labeling and packaging, advertising, promotion, sponsorship, cessation, and curbing illicit trade. In 2016, an independent expert group concluded its impact assessment of the WHO FCTC in the first decade of the treaty. They concluded that the WHO FCTC has led to significant increases in the implementation of tobacco control measures, and that when implemented, key demand-reduction measures of the treaty had led to a significant decline in smoking prevalence ${ }^{1-3}$.

The second most extensive population-level effort in tobacco control is the European Union's (EU) 2014 Tobacco Products Directive (TPD) (2014/40/ EU). The 2014 EU TPD updated existing regulations from the previous EU TPD of 2001 (2001/EC/37), under which all EU Member States (MS) had to meet minimum standards on a number of tobacco control regulatory issues including, but not limited to, tobacco labelling, product design and packaging restrictions, ingredient and emission reporting for tobacco, roll-your-own, smokeless tobacco products, e-cigarettes, novel tobacco products, waterpipe tobacco and herbal smoking products with an implementation date of 20 May 2016, with some regulations allowing for implementation as late as May 2017.

In order to assess the impact of these two large tobacco control efforts at a population level, the EUREST-PLUS Project European Regulatory Science on Tobacco: Policy Implementation to Reduce Lung. Disease, aims to monitor and evaluate the updated 2014 EU TPD, and its associated implementing acts, within the context of WHO FCTC ratification at a European level. The main objective of the EURESTPLUS Project is to monitor and evaluate the impact of the implementing acts of the EU TPD and assess these within the context of WHO FCTC ratification at a European level. A key aspect of the project is the ITC 6 European Country (6E) Surveys-a cohort survey evaluating the psychosocial and behavioural impact of the implementation of the EU TPD Articles and policies outlined in the WHO FCTC among a cohort of smokers in six EU MS . $^{4}$

The present work describes the conceptual framework and methods used by the ITC 6 European Country (6E) Survey to evaluate the regulations of the EU TPD.

\section{METHODS}

Evaluation of tobacco control policies and regulations Population-level interventions such as policies and regulations cannot be evaluated through randomized experiments. However, there are important methodological and design strategies that can be used to increase the internal validity of non-experimental/ observational studies to evaluate the impact of policies, these are: 1) a cohort design in which individuals are measured on the same key outcome variables over time, critically before and after the introduction of the policy (a pre-post design); 2) a quasi-experimental design (i.e. 'natural experiments', or in the language of economists, a 'difference-in-difference design'), in which one group exposed to a policy is compared to a group that has not been exposed, i.e. a pre-post design with control groups (such as other countries) ${ }^{5,6} ; 3$ ) the measurement of appropriate policy-specific variables ('proximal variables') that are conceptually close to the policy being evaluated and less likely to be affected by other factors.

The International Tobacco Control Policy Evaluation (ITC) Project, created in 2002, is an international evidence system that has evaluated WHO FCTC policies across 29 countries, covering over half of the world's population and over two-thirds of the world's tobacco users. The ITC Project was the first, and is still the only, international research program with a focus on WHO FCTC impact evaluation.

The ITC Project incorporates all three strategies in its research design. These three innovative strategies, with the inclusion of other explanatory variables (covariates), include design and content features that are unparalleled in the study of population-level interventions and produce a research design with the potential to make strong inferences about policy impact $^{7}$. Evaluation studies conducted by the ITC Project have provided a large body of evidence on the effectiveness of WHO FCTC policies including price/ tax policies ${ }^{8,9}$, graphic health warnings ${ }^{10-12}$, elimination of 'light/mild' brand descriptors ${ }^{13}$, comprehensive smoke-free laws ${ }^{14-18}$, advertising/promotion bans ${ }^{19}$, impact of plain packaging on effectiveness of health warnings ${ }^{20}$, and cessation policies ${ }^{21}$.

\section{Conceptual framework of the ITC Surveys}

The ITC Project is founded on a strong theory-driven conceptual framework that provides the context for 
the survey content, hypotheses, and data analysis ${ }^{722-24}$. The ITC Conceptual Model is presented in Figure 1:

1. Policies are seen as affecting a variety of psychosocial and behavioral variables. The most immediate effects are those on the policy-specific variables (proximal variables), the variables most connected with the policy itself. Changing from text-only warnings to larger graphic warnings should increase the salience of warnings, as well as other variables that are among the objectives of warnings, e.g. more frequent thoughts about the harms of smoking and about quitting; price should affect the perceived cost of cigarettes (e.g. belief that cigarettes have become too expensive). These effects on the policy-specific variables are the first step in the causal chain. In addition to the convergent validity that multiple measures of impact of a given policy provide, their specificity to a single policy offers the important tool of discriminant validity for distinguishing impact of a single policy in the context of other active policies. For example, the measure 'warnings make me think about the health risks of smoking' was specifically designed to measure changes in health warnings, and not for smoke-free laws or price increases. This specificity allows for greater internal validity in evaluating impact of multiple policies ${ }^{7}$.

2. Psychosocial mediators (distal variables) are the next step in the causal chain. They are known to predict smoking behavior and quitting, and come from well-known psychosocial health behavior models, e.g. Theory of Planned Behavior ${ }^{25}$, Social Cognitive Theory ${ }^{26}$, the Health Belief Model $^{27}$, and Protection Motivation Theory ${ }^{28}$. Policies affect these variables indirectly via effects on policyspecific variables.

3. Outcome variables include beneficial outcomes (e.g. quitting) but also responses that may not lead to the intended public health benefits, e.g. after a tax increase, smokers may switch to cheaper brands, which confers no public health benefit.

The ITC Conceptual Model is thus a causal chain model. In the domain of warnings, if switching to graphic warnings leads to increased quitting ${ }^{29}$, this occurs by first increasing the warning-specific variables (e.g. extent to which warnings make the smoker think about risk). Those changes in the warning-specific variables then lead to changes in psychosocial mediators (e.g. quit intentions). The third step is from those mediators to behavioral outcomes such as quit attempts. Thus, policy affects behavior because it causes changes in the policy specific variables, which in turn cause changes in the psychosocial mediators. Thus, we seek to understand how and why policies have impact, not just whether they have impact.

Figure 1. The ITC Conceptual Model used in the construction of the ITC Surveys ${ }^{22}$

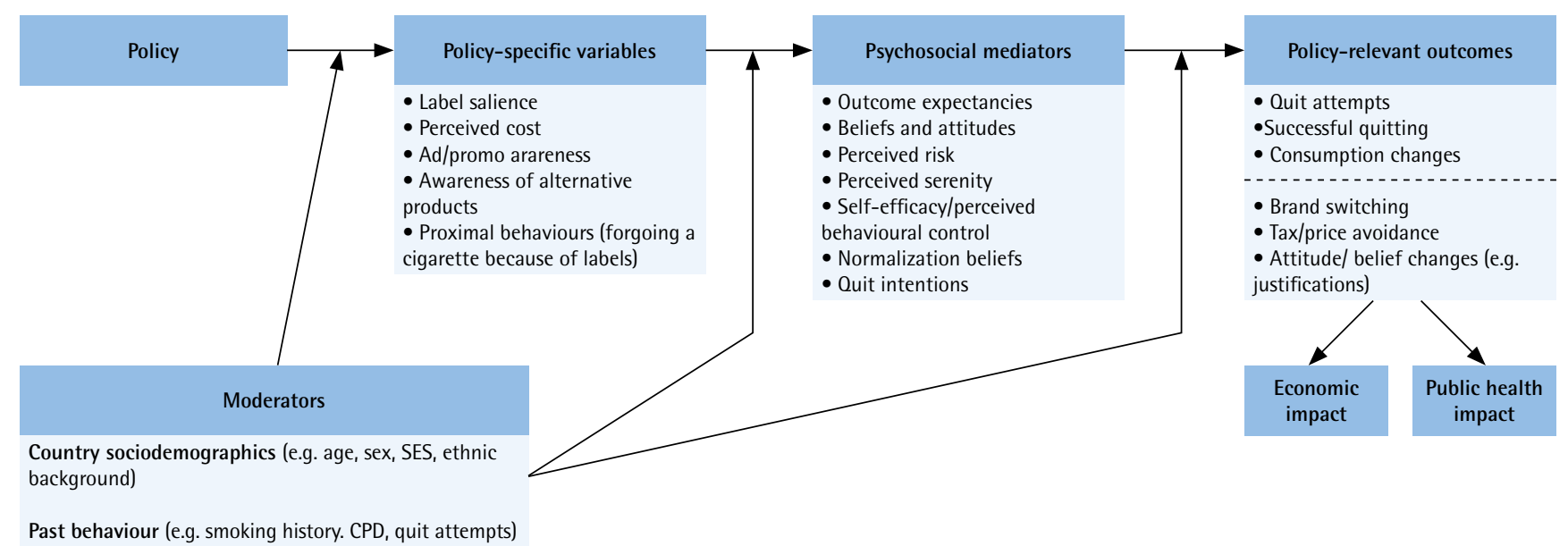

Personality (e.g. time perspective)

Psychological state (e.g. stress)

Potential exposure to policy (e.g. employment status) 
The cohort design allows us some ability to test the causal chain of effects depicted in the model through mediational analysis; whereas a repeat cross-sectional design does not ${ }^{7,22}$. ITC researchers have used structural equation modeling to validate the mediational model in various ITC countries for health warnings ${ }^{30}$ and smoke-free laws ${ }^{18}$.

The ITC Six European Country (6E) Survey is a multi-wave European-focused cohort study with the purpose of measuring the direct and indirect impacts of the EU TPD, and policies of the WHO FCTC. The six countries participating in the ITC 6E Survey are Germany, Greece, Hungary, Poland, Romania, and Spain.

We now describe the methods of data collection and recruitment, the sample sizes and sampling designs, sampling protocols, constructions of survey weights, and survey outcome rates. More detailed information on these topics is available in the ITC 6E Survey Technical Report ${ }^{31}$.

\section{Sample design and features}

The ITC 6E Survey was designed to produce nationally representative samples in each of the six project countries, with a sample size of 1000 smokers from each country in Wave 1. Respondents would qualify if they were of age 18 years or older, smoked at least monthly, and smoked over 100 cigarettes in their lifetime. All interviews were conducted face-to-face using a computer (tablet)-assisted personal interview (CAPI) mode. Survey respondents were interviewed between 18 June and 16 September 2016. In each country, the target number of respondents was either met or exceeded by a few (Table 1).

\section{Sample selection}

The probability sample in each country was chosen by dividing each country into major geographic regions, i.e. Nomenclature of Territorial Units for Statistics or Nomenclature des unités territoriales statistiques, which is a geocode standard for referencing subdivisions of EU countries (NUTS2 regions; NUTS1 in Germany). The Greek Islands in the Ionian Sea, The Northern Aegean Sea, and Southern Aegean Sea were excluded from the sample in Greece, as were the Canary Islands, Ceuta, and Melilla from Spain. The geographic strata were made up of the NUTS regions crossed with the degree of urbanization, that is whether the region
Table 1. Total number of interviewed respondents by country

$\begin{array}{lc}\text { Country } & \text { Sample sine } \\ \text { Germany } & 1003 \\ \text { Greece } & 1000 \\ \text { Hungary } & 1000 \\ \text { Poland } & 1006 \\ \text { Romania } & 1001 \\ \text { Spain } & 1001 \\ \text { Overall } & 6011\end{array}$

was urban, semi urban-rural, or rural. The strata were conceptually conceived to be a grouping of clusters, each the size of an enumeration area. The numbers of clusters to be sampled were allocated to strata approximately proportionally to the size of the population of age 18 years or older. The objective in the design was to sample 100 random clusters within each country, with at least two clusters in each stratum. Within each cluster, 10 adult smokers would be interviewed.

In each cluster, interviewers used a random walk method to select each dwelling to be approached. The tablet program selected a starting point at random using only GPS coordinates, and a random walk path. Following the designated path, interviewers approached every 5 th address. In cases where the address selected contained multiple households, a random unit was selected. Four attempts to make contact were required for each selected dwelling unit before a new address could be selected.

When a household was contacted, a knowledgeable informant was identified to provide the number of male and female smokers aged 18 or older. Using the next birthday method ${ }^{32}$, one male smoker and one female smoker were selected for interview where possible. Should only one smoker of a given gender be available, that person was automatically selected. To ensure the randomness of the sample, no substitutions were allowed within a household, with the exception of replacement of selected individuals who were unavailable for the entirety of the fieldwork period. This process of screening, selection and interviewing continued within a cluster until the required number of smokers were interviewed.

\section{Survey development}

As with all ITC surveys, the ITC 6E Wave 1 Survey was 
designed with the following aims in mind: 1) examine the impact of tobacco control policies, including the EU TPD and the WHO FCTC; 2) compare smoking behaviours and policy impact among the six countries and the wider group of ITC countries; and 3) gather evidence to suggest changes to current tobacco policy. To accomplish these aims, the ITC 6E Survey drew questions from several active ITC surveys that measure smoking-related behaviours, perceptions and attitudes toward quitting, policy-based measurements including questions on warnings, advertisements/ promotion of tobacco products, and more. Due to the expanding scope of tobacco policy and related novel products, questions were added covering products such as electronic cigarettes and vaping devices, as well as heated tobacco devices. Finally, moderators and psychosocial measures were included that provide behavioural context, as well as basic demographic information.

The majority of the survey questions for the 6E Survey had already been used in ITC surveys conducted in other countries; this commonality was helpful to allow for valid cross-country comparisons. There were, however, a number of specific policy issues in the EU TPD that required new questions to be developed. Several teleconferences were held between EUREST-PLUS Project members to discuss, propose, and edit possibilities for appropriate survey questions to measure the impact of EU TPD regulations, as well as to make changes across the survey to be more appropriate for the European context and also across the diversity of the six countries (e.g. to make changes in terminology or phrasing because of differences across countries in how concepts are expressed).

Because of the objective of conducting pre-post evaluation analyses of the EU TPD, it was important that Wave 1 was conducted as early as possible to allow for data collection before the EU TPD was implemented, and that Wave 2 would be conducted after key measures and regulations of the EU TPD had been implemented (Wave 2 was conducted starting in late February 2018), to measure change over time. While the EU TPD compliance date in the declaration was 20 May 2016, some of the provisions of the EU TPD were allowed another year for implementation. The fieldwork of the Wave 1 survey began on 18 June. This meant that some of the EU TPD's impact may have been experienced at the time of the Wave 1 survey. However, some of the provisions of the EU TPD had extended dates for full implementation, so the slight delay in the launching of Wave 1 was not very problematic. Wave 1 stands as an accurate benchmark of the time of implementation, and a strong point of comparison for future waves to measure the potential impact of the EU TPD.

\section{Survey management}

The survey fieldwork for the ITC 6E Survey was managed by Kantar Public in Brussels, one of the partner organizations within the EUREST-PLUS Project. Kantar Public put out a public call for local survey teams within each of the six countries, as required by the EUREST-PLUS funding mechanism, the Horizon 2020 Funding Scheme. The fieldwork was conducted in the 6 European countries by the following agencies: Foerster and Thelen (Germany), Metron Analysis (Greece), Kantar TNS Hoffman (Hungary), Kantar TNS Polska (Poland), Curs (Romania), and Kantar TNS Spain (Spain).

Kantar Public oversaw the translation of all survey materials. With final scripts completed in English, the translation into the six national languages was handled in-house. For each language, the questionnaire was translated first by an independent translator, at which point the survey went through a two-stage revision period: first, via a second independent proof-reader; and second, by the national agency project manager. Upon completion, the translated survey was sent to the respective country team members of the ITC $6 \mathrm{E}$ Country Project to ensure accuracy.

Kantar Public was also responsible for overseeing the training of the local fieldwork agencies listed above. Representatives from Kantar trained local team members on the use of Kantar's Computer Assisted Personal Interview (CAPI) survey software, ensuring that fieldwork operators could follow the fieldwork procedures, both in sampling and in interviewing respondents.

The protocol at each approached household followed seven basic steps: A basic introduction; the administration of the household screener to a knowledgeable individual - a short survey to ensure that qualified respondents resided within the household; an information and consent procedure 
for selected respondents; the administration of an individual screener to the selected respondents to ensure qualification; the administration of the full ITC 6E Survey; and a thank you including remuneration for the respondents' time. Incentive amounts were established in consultation with local survey teams and based on best practices for each country ( $10 €$ in Germany, Hungary and Poland, $7 €$ in Romania, $5 €$ in Greece, and $3 €$ in Spain), see Table 2.

Due to the international nature of the project, fieldwork timeframes varied from country to country both in start and completion dates (Table 3 ). Fieldwork in Greece was suspended for one week (12 August to 19 August) to accommodate the summer vacation period within the country, as a significant amount of the population was unavailable during this time, it was important to postpone fieldwork to ensure accurate representativeness.

The survey protocols and all materials, including the survey questionnaires, were cleared for ethics by the Office of Research Ethics, University of Waterloo, Canada and by local ethics boards in the participating countries. Written consents from respondents were obtained.

\section{Quality control}

Monitoring the fieldwork to ensure quality control was achieved through multiple checks. First,

Table 2. Respondent remuneration by country

$\begin{array}{lc}\text { Coumntry } & \text { Incentive (C) } \\ \text { Germany } & 10 \\ \text { Greece } & 5 \\ \text { Hungary } & 10 \\ \text { Poland } & 10 \\ \text { Romania } & 7 \\ \text { Spain } & 3\end{array}$

Table 3. Fieldwork start and end dates (all in 2018)

\begin{tabular}{l|c|c} 
Country & Start date & End date \\
Germany & 15 June & 30 August \\
Greece & 16 June & 12 September \\
Hungary & 22 June & 20 July \\
Poland & 25 June & 21 August \\
Romania & 25 June & 8 August \\
Spain & 23 June & 21 July
\end{tabular}

fieldwork in all countries was managed centrally by Kantar Public in Brussels. Field data were transmitted via NFIELD software to Kantar, allowing them to monitor and assess progress in real time. Second, Fieldwork Progress Reports were provided by the Project Manager to Kantar Public, who then sent weekly updates to the University of Waterloo, Canada, for additional monitoring. Third, Field Supervisors were appointed and charged with supervising interviewers and assisting them with any questions or issues related to fieldwork protocol. Finally, at the local level, $10 \%$ back checks were conducted on interviews conducted within a week of completion. Here, checks on coding and comparisons between raw samples of data and national populations were conducted ensuring item response was $100 \%$ within the completed surveys.

\section{Data protection}

The database of the survey responses are identified only by a unique ID number, without participants' identifiable information. All data analyses were conducted on the de-identified data.

\section{External data sharing}

Data from this project are available to approved researchers starting two years after the date of issuance of cleaned data sets by the ITC Data Management Centre at the University of Waterloo. Researchers interested in using ITC $6 \mathrm{E}$ data are required to apply for approval by submitting an International Tobacco Control Data Repository (ITCDR) request application and subsequently to sign an ITCDR Data Usage Agreement.

\section{Survey weights}

It is standard among ITC datasets to construct weights that correct and adjust for sample misrepresentation due to factors such as unequal sampling probabilities, frame error, and non-responses. It is also desirable to improve the precision of the datasets estimates through the application of information available via auxiliary sources, for instance sociodemographic benchmarks ${ }^{33}$.

In order to compute sampling weights for each smoker participating in the survey, first, each respondent was assigned an initial weight equal to the reciprocal of their probability of being selected 
within their household. Second, the probability of the respondent's inclusion within the stratum was estimated. As the design for sampling dwellings was a random walk procedure, the probability of inclusion of a dwelling or household is proportional to the number of random walks in which the household would be sampled. Since these numbers were unknown, the inclusion probability of a household was taken to be approximately equal within each stratum, and thus the inclusion probability of an individual within the stratum was taken to be proportional to the selection probability within the household. Third, a post-stratification adjustment based on estimated smoker prevalences from Eurobarometer 2014 was performed to calibrate weights among stratum, sex, and age groups. Finally, for analytic use, particularly those involving comparisons across the six countries, as well as with other ITC country datasets, the weights were rescaled to have a mean equal to 1 in each country. To account for the complexity of the sampling design in the estimation of standard errors and computation of confidence intervals, bootstrap weights were provided based on the Rao and $\mathrm{Wu}$ $(1998)^{34}$ technique.

\section{Survey outcome rates}

Table 4 shows the calculation of household and individual response rates and cooperation rates. Household contact rates were high, except in Romania; the household response rates were moderate, being somewhat lower in Germany and Greece, where there were evidently greater proportions of refusals to have eligibility determined. Household and individual cooperation rates were high.

Table 4. Survey outcome rates

\begin{tabular}{|c|c|c|c|c|c|c|}
\hline & Germany & Greece & Hungary & Poland & Romania & Spain \\
\hline $\begin{array}{l}\text { 1. Number of addresses approached/ } \\
\text { attempted }\end{array}$ & 10325 & 3537 & 2754 & 3421 & 4490 & 4114 \\
\hline $\begin{array}{l}\text { 2. Number of addresses where contact } \\
\text { was made }\end{array}$ & 8259 & 2856 & 2404 & 3028 & 2778 & 3853 \\
\hline $\begin{array}{l}\text { 3. Number of contacted addresses with } \\
\text { eligibility determined }\end{array}$ & 3086 & 1295 & 1791 & 2154 & 2065 & 2451 \\
\hline $\begin{array}{l}\text { 4. Number of contacted addresses with } \\
\text { no eligible respondents }\end{array}$ & 1982 & 466 & 972 & 1163 & 1134 & 1509 \\
\hline $\begin{array}{l}\text { 5. Number of contacted addresses with } \\
\text { eligible respondents }\end{array}$ & 1104 & 829 & 819 & 991 & 931 & 942 \\
\hline $\begin{array}{l}\text { 6. Number of addresses with eligible } \\
\text { respondents, members selected }\end{array}$ & 1085 & 823 & 809 & 976 & 911 & 936 \\
\hline $\begin{array}{l}\text { 7. Eligibility rate for households, given } \\
\text { determination of eligibility (5./3.) }\end{array}$ & 0.358 & 0.640 & 0.457 & 0.460 & 0.451 & 0.384 \\
\hline $\begin{array}{l}\text { 8. Estimated eligible households among } \\
\left.\text { attempted ( } 7 .{ }^{*} 1 .\right)\end{array}$ & 3694 & 2264 & 1259 & 1574 & 2024 & 1581 \\
\hline $\begin{array}{l}\text { 9. Number of individuals selected for } \\
\text { interview }\end{array}$ & 1425 & 1116 & 1078 & 1265 & 1254 & 1234 \\
\hline $\begin{array}{l}\text { 10. Number of individual refusals or } \\
\text { break offs }\end{array}$ & 200 & 91 & 64 & 227 & 232 & 219 \\
\hline 11. Number of completed interviews & 1003 & 1000 & 1000 & 1006 & 1003 & 1001 \\
\hline 12. Household contact rate (2./1.) & 0.800 & 0.807 & 0.873 & 0.885 & 0.619 & 0.937 \\
\hline $\begin{array}{l}\text { 13. Household cooperation rate, given } \\
\text { eligible (6./5.) }\end{array}$ & 0.983 & 0.993 & 0.988 & 0.985 & 0.979 & 0.994 \\
\hline 14. Household response rate (6./8.) & 0.294 & 0.363 & 0.642 & 0.620 & 0.450 & 0.592 \\
\hline $\begin{array}{l}\text { 15. Individual cooperation rate } \\
(11 . /(11 .+10 .))\end{array}$ & 0.834 & 0.917 & 0.940 & 0.816 & 0.812 & 0.820 \\
\hline $\begin{array}{l}\text { 16. Individual response rate, given } \\
\text { selection (11./9.) }\end{array}$ & 0.704 & 0.896 & 0.928 & 0.795 & 0.800 & 0.811 \\
\hline
\end{tabular}




\section{DISCUSSION}

Wave 1 of the ITC 6 European Country Survey employed the same conceptual model, as well as identical or functionally similar methods that have been employed across all 29 countries of the ITC Project. This consistency across countries over time allows for strong comparability of the findings from these six EU countries to the three other EU countries participating in the ITC Project (United Kingdom, Netherlands, France) and across the 20 non-European ITC countries. The data from Wave 2, conducted beginning in February 2018, will allow for important pre-post evaluation studies of the EU TPD and other tobacco control measures.

\section{CONCLUSIONS}

The ITC 6 European Country Survey, and the broader EUREST-PLUS Project, holds great potential to provide important evidence regarding the impact of measures designed to reduce tobacco use, a major cause of premature death and disease in Europe and throughout the world.

\section{*EUREST-PLUS consortium members:}

European Network on Smoking and Tobacco Prevention (ENSP), Belgium: Constantine I. Vardavas, Andrea Glahn, Christina N. Kyriakos, Dominick Nguyen, Cornel Radu-Loghin, Polina Starchenko

University of Crete (UoC), Greece: Aristidis Tsatsakis, Charis Girvalaki, Chryssi Igoumenaki, Katerina Nikitara, Sophia Papadakis, Aikaterini Papathanasaki, Manolis Tzatzarakis, Alexander I. Vardavas

Kantar Public (TNS), Belgium: Nicolas Bécuwe, Lavinia Deaconu, Sophie Goudet, Christopher Hanley, Oscar Rivière

Smoking or Health Hungarian Foundation (SHHF), Hungary: Tibor Demjén, Judit Kiss, Piroska A. Kovacs

Catalan Institut of Oncology (ICO); Bellvitge Biomedical Research Institute (IDIBELL), Spain: Esteve Fernández, Yolanda Castellano, Marcela Fu, Sarah 0. Nogueira, Olena Tigova

Kings College London (KCL), United Kingdom: Ann McNeill, Katherine East, Sara C. Hitchman

Cancer Prevention Unit and WHO Collaborating Centre for Tobacco Control, German Cancer Research Center (DKFZ), Germany: Ute Mons, Sarah Kahnert

University of Athens (UoA), Greece: Yannis Tountas, Panagiotis Behrakis, Filippos T. Filippidis, Christina Gratziou, Paraskevi Katsaounou, Theodosia Peleki, Ioanna Petroulia, Chara Tzavara

Aer Pur Romania, Romania: Antigona C. Trofor, Marius Eremia, Lucia Lotrean, Florin Mihaltan

European Respiratory Society (ERS), Switzerland; Goethe University Frankfurt, Germany: Gernot Rohde, Tamaki Asano, Claudia Cichon, Amy Far, Céline Genton, Melanie Jessner, Linnea Hedman, Christer Janson, Ann Lindberg, Beth Maguire, Sofia Ravara, Valérie Vaccaro, Brian Ward

Maastricht University, the Netherlands: Marc Willemsen, Hein de Vries, Karin Hummel, Gera E. Nagelhout

Health Promotion Foundation (HPF), Poland: Witold A. Zatoński, Aleksandra Herbeć, Kinga Janik-Koncewicz, Krzysztof Przewoźniak, Mateusz Zatoński University of Waterloo (UW); Ontario Institute for Cancer Research, Canada: Geoffrey T. Fong, Thomas K. Agar, Pete Driezen, Shannon Gravely, Anne C. K. Quah, Mary E. Thompson

\section{REFERENCES}

1. Chung-Hall J, Craig L, Gravely S, Sansone N, Fong GT. Impact of the WHO FCTC over the first decade: A global evidence review prepared for the Impact Assessment Expert Group. Tob Control. 2018. doi:10.1136/tobaccocontrol-2018-054389

2. Conference of the Parties to the WHO Framework Convention on Tobacco Control. FCTC/COP/7/6. Impact assessment of the WHO FCTC: Report by the Expert Group. Geneva: WHO; 2016. http://www.who.int/fctc/ cop/cop7/FCTC_COP_7_6_EN.pdf. Accessed November 2,2018 .

3. Gravely S, Giovino GA, Craig LV, et al. Implementation of key demand-reduction measures of the WHO Framework Convention on Tobacco Control and change in smoking prevalence in 126 countries: an association study. Lancet Public Health. 2017;2(4):e166-e174. doi:10.1016/S2468-2667(17)30045-2

4. Vardavas CI, Bécuwe N, Demjén T, et al. Study Protocol of European Regulatory Science on Tobacco (EURESTPLUS): Policy implementation to reduce lung disease. Tobacco Induced Diseases. 2018;16(Suppl 2:A2). doi:10.18332/tid/93305

5. Cook T, Campbell DT. Quasi-experimentation: Design $\&$ analysis issues for field settings. Boston: HoughtonMifflin; 1979.

6. Shadish WR, Cook TD, Campbell DT. Experimental and quasi-experimental designs for generalized causal inference. 2nd ed. Boston: Houghton-Mifflin; 2002.

7. International Agency for Research on Cancer (IARC). Methods for Evaluating Tobacco Control Policies: IARC Handbook of Cancer Prevention. Vol 12. Lyon, FR: WHO Press; 2008.

8. Azagba S, Burhoo P, Chaloupka FJ, Fong GT. Effect of cigarette tax increase in combination with mass media campaign on smoking behavior in Mauritius: Findings from the ITC Mauritius Survey. Tob Control. 2015;24(Suppl 3):iii71-iii75. doi:10.1136/tobaccocontrol-2014-052096

9. Li Q, Hu T, Mao ZZ, et al. When a tax increase fails as a tobacco control policy: The ITC China Project evaluation of the 2009 cigarette tax increase in China. Tob Control. 2012;21(3):381. doi:10.1136/tobaccocontrol-2011-050111

10. Elton-Marshall T, Xu SS, Meng G, et al. The lower effectiveness of text-only health warnings in China compared to pictorial warnings in Malaysia: Findings from the ITC Project. Tob Control. 2015;24(Suppl 4):iv6-iv13. doi:10.1136/tobaccocontrol-2015-052616

11. Gravely S, Fong GT, Driezen P, et al. The impact of the 2009/2010 enhancement of cigarette health warning labels in Uruguay: longitudinal findings from the International Tobacco Control (ITC) Uruguay Survey. Tob Control. 2016;25(1):89-95. doi:10.1136/tobaccocontrol-2014-051742

12. Green AC, Kaai SC, Fong GT, Driezen P, Quah ACK, 
Burhoo P. Investigating the effectiveness of pictorial health warnings in Mauritius: findings from the ITC Mauritius survey. Nicotine Tob Res. 2014;16(9):12401247. doi:10.1093/ntr/ntu062

13. Borland R, Fong GT, Yong HH, et al. What happened to smokers' beliefs about light cigarettes when "Light/ Mild" brand descriptors were banned in the UK? Findings from the International Tobacco Control (ITC) Four Country Survey. Tob Control. 2008;17(4):256-262. doi:10.1136/tc.2007.023812

14. Fong GT, Hyland A, Borland R, et al. Reductions in tobacco smoke pollution and increases in support for smoke-free public places following the implementation of comprehensive smoke-free workplace legislation in the Republic of Ireland: Findings from the ITC Ireland/ UK Survey. Tob Control. 2006;15(Suppl III):iii51-iii58. doi:10.1136/tc.2005.013649

15. Fong GT, Craig LV, Guignard R, et al. Evaluating the effectiveness of France's indoor smoke-free law 1 year and 5 years after its implementation: Findings from the ITC France Survey. PLoS ONE. 2013;8(6):e66692. doi:10.1371/journal.pone.0066692

16. Mons U, Nagelhout GE, Allwright S, et al. Impact of national smoke-free legislation on home smoking bans: Findings from the International Tobacco Control (ITC) Policy Evaluation Project Europe Surveys. Tob Control. 2013;22(e1):e2-e9. doi:10.1136/tobaccocontrol-2011-050131

17. Nagelhout GE, de Vries H, Allwright S, et al. Comparative impact of smoke-free legislation on smoking cessation in three European countries. Eur J Public Health. 2012;22(Suppl 1):4-9. doi:10.1093/eurpub/ckr203

18. Nagelhout GE, de Vries H, Fong GT, et al. Pathways of change explaining the effect of smoke-free legislation on smoking cessation in The Netherlands. An application of the international tobacco control conceptual model. Nicotine Tob Res. 2012;14(12):1474-1482. doi:10.1093/ntr/nts081

19. Harris F, MacKintosh AM, Anderson S, et al. Effects of the 2003 advertising/promotion ban in the United Kingdom on awareness of tobacco marketing: Findings from the International Tobacco Control (ITC) Four Country Survey. Tob Control. 2006;15(Suppl 3):iii16iii33. doi:10.1136/tc.2005.013110

20. Yong HH, Borland R, Hammond D, Thrasher JF, Gummings KM, Fong GT. Smokers' reactions to the new larger health warning labels on plain cigarette packs in Australia: Findings from the ITC Australia Project. Tob Control. 2016;25(2):181-187. doi:10.1136/tobaccocontrol-2014-051979

21. Gibson JE, Murray RL, Borland R, et al. The impact of the United Kingdom's national smoking cessation strategy on quit attempts and use of cessation services: Findings from the International Tobacco Control Four Country Survey. Nicotine Tob Res. 2010;12(Suppl 1):64-71. doi:10.1093/ntr/ntq119
22. Fong GT, Cummings KM, Borland R, et al. The conceptual framework of the International Tobacco Control (ITC) Policy Evaluation Project. Tob Control. 2006;15(Suppl 3):iii3-iii11. doi:10.1136/tc.2005.015438

23. Fong GT, Cummings KM, Shopland DR, for the ITC Collaboration. Building the evidence base for effective tobacco control policies: The International Tobacco Control Policy Evaluation Project (the ITC Project). Tob Control. 2006;15(Suppl 3):iii1-iii2. doi:10.1136/tc.2006.017244

24. Thompson ME, Fong GT, Hammond D, et al. Methods of the International Tobacco Control (ITC) Four Country Survey. Tob Control. 2006;15(Suppl 3):iii12-iii 18. doi:10.1136/tc.2005.013870

25. Ajzen I. The theory of planned behavior. Organizational Behav Hum Decision Proc. 1991;50(2):179-211. doi:10.1016/0749-5978(91)90020-T

26. Bandura A. Social foundations of thought and action: A social cognitive theory. Englewood Cliffs, NJ: PrenticeHall; 1986.

27. Becker MH. The health belief model and personal health behavior. Thorofare, NJ: Charles B. Slack; 1974.

28. Rogers R, Prentice-Dunn S. Protection Motivation Theory. New York: Plenum; 1997:113-132.

29. Borland R, Yong HH, Wilson N, et al. How reactions to cigarette packet health warnings influence quitting: findings from the ITC FourCountry Survey. Addiction. 2009;104(4):669-675. doi:10.1111/j.1360-0443.2009.02508.x

30. Yong HH, Borland R, Thrasher JF, et al. Mediational pathways of the impact of cigarette warning labels on quit attempts. Health Psychol. 2014;33(11):1410-1420. doi:10.1037/hea0000056

31. ITC Project. ITC 6 European Country Wave 1 (2016) Technical Report. Waterloo, Canada: University of Waterloo, and Belgium: European Network on Smoking and Tobacco Prevention; 2017.

32. Binson D, Catania JA. Random selection in a national telephone survey: a comparison of the Kish, nextbirthday and last-birthday methods. J Official Stat. 2000;16(1):53-60.

33. Levy PS, Lemeshow S. Sampling of Populations: Methods and Applications. 4th ed. Hoboken, NJ: John Wiley \& Sons; 2008.

34. Rao JNK, Wu CFJ. Resampling inference with complex survey data. J Am Stat Assoc. 1988;83:231-241. doi:10.2307/2288945 
CONFLICTS OF INTEREST

The authors declare that they have no competing interests, financial or otherwise, related to the current work. N. Bécuwe reports grants from Kantar Public Brussels, during the conduct of the study. K. Przewoźniak reports grants and personal fees from the Polish League Against Cancer, outside the submitted work. C. I. Vardavas reports that he is the Strategic Development Editor of TID and that there are no conflicts of interest with this current work. The rest of the authors have also completed and submitted an ICMJE form for disclosure of potential conflicts of interest.

FUNDING

This project has received funding from the European Union's Horizon 2020 research and innovation programme under grant agreement No 681109 (C. I. V.) and the University of Waterloo (G. T. F.). Additional support was provided to the University of Waterloo by the Canadian Institutes of Health Research (FDN-148477). G. T. F. was supported by a Senior Investigator Grant from the Ontario Institute for Cancer Research. E. F. , M. F. , O. T. , and Y. C. are supported by the Ministry of Universities and Research, Government of Catalonia (2017SGR319). E. F. was supported by the Instituto de Salud Carlos III, Government of Spain (INT16/00211 and INT17/00103), co-funded by the European Regional Development Fund (FEDER).

PROVENANCE AND PEER REVIEW

Commissioned; internally peer reviewed. 